\title{
High resolution lunar gravity anomaly map from the lunar prospector line-of-sight acceleration data
}

\author{
Takayuki Sugano ${ }^{1}$ and Kosuke Heki ${ }^{1}$ \\ ${ }^{1}$ Department of Astronomical Science, Graduate University for Advanced Studies, \\ 2-12 Hoshigaoka, Mizusawa, Iwate 023-0861, Japan
}

(Received October 17, 2003; Revised December 24, 2003; Accepted December 24, 2003)

\begin{abstract}
Study of the lunar gravity anomaly has not been straightforward since direct tracking data of lunar satellites are available only at its nearside. In such a case, direct inversion of the line-of-sight acceleration data into surface mass distribution has several merits, e.g., (1) high resolution can be attained without relying on artificial constraints, (2) short computation time by estimating regional parameter sets stepwise. After confirming the validity of the method using synthesized data, we processed the line-of-sight acceleration data of the Lunar Prospector extended low-altitude mission. The obtained gravity anomaly map of the lunar nearside has resolution as high as $0.8^{\circ} \times 0.8^{\circ}$, equivalent to 225th degree/order of spherical harmonics, with less spurious signatures than past studies. To take advantage of the high resolution, we calculate mass deficits for 92 medium-sized craters (50-300 km in diameter), and confirmed that they are nearly proportional to 2.5 power of crater diameter.
\end{abstract}

Key words: Moon, gravity anomaly, LOS, free air, lunar crater, mass deficit.

\section{Introduction}

Gravity field data of terrestrial planets and satellites are rich in information on their interiors. The longestwavelength components, e.g. $J_{2}$ and $C_{22}$, are used to estimate their moments of inertia (Williams et al., 1996). On the other hand, short-wavelength components provide information on physical properties of relatively shallow parts. For example, compensation depth (or lithospheric thickness), inferred by comparing the topography and gravity anomalies in various wavelengths, is useful in constraining their thermal histories. Gravity field studies of the Moon began in 1966 with the satellite Luna 10, followed by Lunar Orbiters I-V and Apollo 15, 16 sub-satellites in 1970's. Several lunar gravity models, expressed as the spherical harmonic expansion of the gravity potential, have been made by analysing tracking data of lunar satellites (e.g. Bills and Ferrari, 1980). The accuracy of such models was dramatically improved by the recent two lunar satellites, Clementine (Lemoine et al., 1997) and Lunar Prospector (LP) in 1990's.

The LP was launched on January 7, 1998 (Binder, 1998). After finishing the 1-year nominal mission at the $100 \mathrm{~km}$ altitude polar orbit, 6-months long extended mission was carried out at the average height of $30 \mathrm{~km}$. Tracking data for such low orbits are rich in information on short-wavelength gravity features of the Moon. The two-way Doppler tracking data of LP during the nominal mission yielded the LP75G lunar gravity field model, which consists of the Stokes' coefficients complete to 75 th degree and order (Konopliv et al., 1998). Using the low-altitude extended mission tracking data, Konopliv et al. (2001) recently reported a higher-

Copy right (c) The Society of Geomagnetism and Earth, Planetary and Space Sciences (SGEPSS); The Seismological Society of Japan; The Volcanological Society of Japan; The Geodetic Society of Japan; The Japanese Society for Planetary Sciences; TERRA PUB resolution model called LP165P, in which they performed the inversion calculation in multiple steps. This was, however, only partly successful due to the lack of direct tracking data of the lunar farside. Their model is reliable up to 110th degree in the nearside and 60th degree in the farside (Konopliv et al., 2001); the gravity anomaly map has many spurious linear features when drawn using all the coefficients (Fig. 1(a)). It is thus difficult to take advantage of the short-wavelength gravity information originally included in the tracking data using this gravity model.

Line-of-sight (LOS) acceleration data of the LP extended mission are available at the Planetary Data System (PDS) Geosciences Node (http://wwwpds.wustl.edu). They carry short-wavelength lunar gravitational field information intact, and are suitable to investigate fine structures of the lunar gravity field. In this paper we try to make a high resolution lunar gravity anomaly map using this data set, in a way to circumvent problems arising from the non-uniform distribution of the tracking data. Then we estimate mass deficits associated with medium-sized impact craters from the high resolution gravity anomaly map.

\section{Analysis Method of LOS Data \\ 2.1 The LOS acceleration data}

The LP satellite was tracked with the NASA/JPL Deep Space Network (DSN). Two-way Doppler data were taken every 10-second interval, which corresponds to $\sim 15 \mathrm{~km}$ distance at the lunar surface. The accuracy of the tracking data is about $0.3 \mathrm{~mm} \mathrm{~s}^{-1}$ (Konopliv et al., 2001). Hence the accuracy of LOS acceleration is considered to be around $0.04 \mathrm{~mm} \mathrm{~s}^{-2}$ (4 mgal).

The two-way Doppler tracking data from the DSN stations are processed with the software package LUNRES, where the LOS acceleration data were obtained by the following 

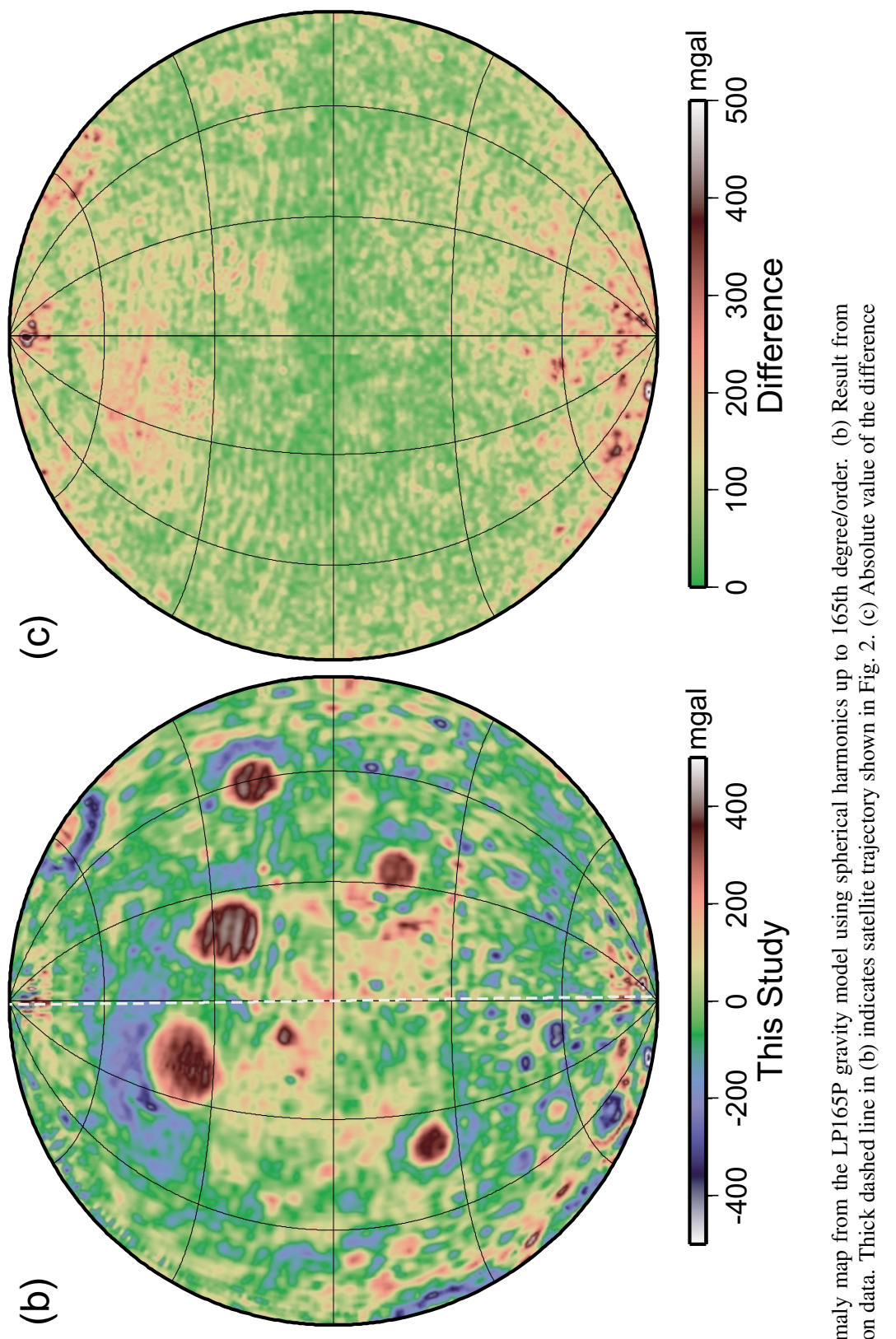

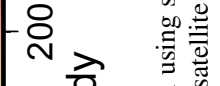

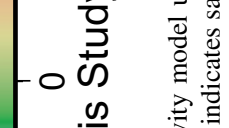

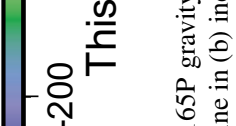

:

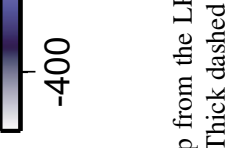
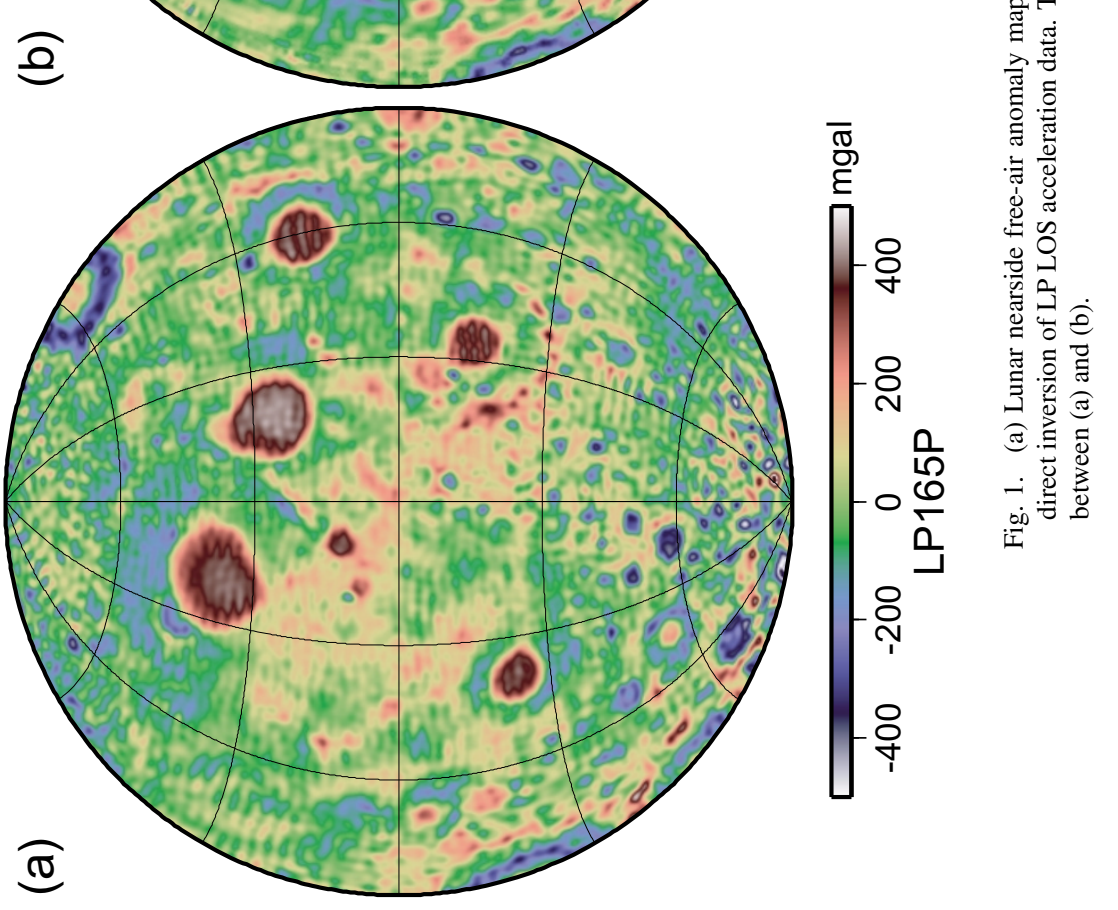

- 웅

- 웅 
(a)

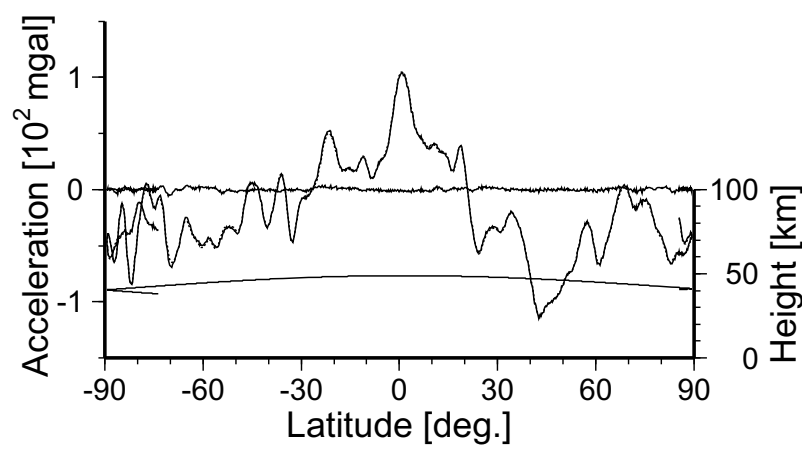

(b)

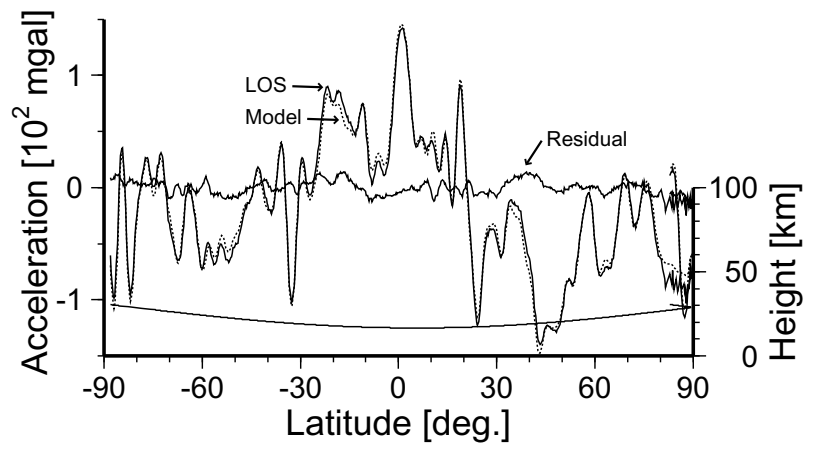

Fig. 2. Example of the LOS acceleration profiles along the longitude $\sim 0^{\circ}$ at relatively high (a) and low (b) altitudes. The amplitude of "residual" becomes large when the satellite altitude is low.

procedure (see Simpson, 1999, for detail). First, the LOS velocities based on the satellite orbits and the lunar gravity model (LP100J) are subtracted from the observed Doppler data. These velocity residuals contain information on shortwavelength gravity fields not represented by spherical harmonics with degrees and orders up to 100 . Second, the time series of residuals are modeled using cubic polynomial spline functions so that accelerations can be obtained as their temporal differentiation. Finally, such residual accelerations were added back to accelerations at the satellite positions predicted by the LP100J model in order to obtain the total LOS acceleration. The processed LOS data are available at the PDS Geosciences Node web site as described above. We downloaded the LOS data product from PDS web site, and used them for the present study. Examples of an LOS acceleration profile along longitude $\sim 0^{\circ}$ are shown in Fig. 2 (The satellite trajectory is plotted in Fig. 1(b)).

\subsection{Inversion method}

The bulk of lunar gravity fields can be approximated by those due to a point mass at the lunar center-of-gravity, which we call the "reference field". This is different from the Earth's reference gravity field in several aspects. First, it is calculated assuming a sphere instead of an ellipsoid because the lunar equatorial bulge is much smaller than the Earth. Secondly, it does not include centrifugal forces coming from the lunar spin. This is because we measure the lunar gravity field from the orbit rather than on the surface. We assume that excess masses (or mass deficits), responsible for the anomalous gravity (difference from the reference field), are condensed in a thin layer on the lunar reference surface (a sphere with a radius $1,738 \mathrm{~km}$ ). This is untrue for long wavelength components such as $J_{2}$ and $C_{22}$, and the results would be somewhat biased in these components. This, however, does not affect our purpose to study short-wavelength gravity anomalies. This approximation also assumes that satellite altitudes are much larger than vertical scales of lunar topographic features. Validity of this assumption will be examined by performing inversion calculations for synthesized data in Section 2.3.

We divide the lunar surface into $20^{\circ} \times 20^{\circ}$ "large blocks". They are subdivided into $0.8^{\circ} \times 0.8^{\circ}$ "small blocks" (about $25 \mathrm{~km} \times 25 \mathrm{~km}$ ). The "large block" is a unit of leastsquare estimation runs, and the "small block" corresponds to a parameter (point mass at the center of the block) to be estimated. The east-west extent of small blocks are increased by a factor of $1 / \cos ($ latitude) in order to keep physical sizes of small blocks uniform.

Assuming that anomalous gravity fields at satellite positions are the sum of the gravitational pulls of individual small blocks, we estimated their masses so that they explain the observed LOS acceleration best in a least-squares sense. The observation equation, based on the simple Newton's theory, is

$$
a_{l o s i}=\sum_{j=1}^{M} G \frac{m_{j}}{r_{i j}^{2}} \cos \theta_{i j},
$$

where $M$ is the number of small blocks within a large block, $G$ is the universal gravitational constant, $m_{j}$ is the mass anomaly at the $j$ th small block, $r_{i j}$ is the distance between the satellite and the $j$ th small block, $\theta_{i j}$ is the angle between gravitational acceleration from the $j$ th small block and the LOS vector at the observation time.

The LOS direction is almost stationary but slightly changes in time due to (1) optical libration of the Moon, (2) parallax due to the Earth's spin, and (3) parallax due to the orbital motion of the satellite. Here we evaluate the influences of the three factors. Due to the optical libration, the apparent position of the Earth in the lunar sky changes in monthly periods by about $\pm 5^{\circ}$ (North, 2000). This can be calculated using the Earth's direction in the moon-fixed coordinate given in the header part of the LOS data files. Roughly speaking, the LOS acceleration is the projection of gravitational acceleration of the Moon $g_{\text {moon }}$ (about $162 \mathrm{gal}$ ) onto the LOS direction, i.e., $g_{\text {moon }} \cos \delta$ where $\delta$ is the angle between LOS acceleration and the lunar vertical. Then the effect of the error in LOS is $-g_{\text {moon }} \Delta \delta \sin \delta$. Hence the error does not exceed $g_{\text {moon }} \Delta \delta$. For the factor (1), the error become $\pm 14.1 \mathrm{mgal}$, a few times as large as the LOS acceleration accuracy. However, the factors (2) and (3) are within $\pm 0.75^{\circ}( \pm 2.19 \mathrm{mgal})$ and $\pm 0.25^{\circ}( \pm 0.71 \mathrm{mgal})$, respectively. Therefore, we took account of only the factor (1) in our study.

An element of the Jacobian matrix $\mathbf{A}$ is calculated as,

$$
A_{i j}=\frac{\partial a_{l o s i}}{\partial m_{j}}=G \frac{1}{r_{i j}^{2}} \cos \theta_{i j} .
$$


(a) $10-20 \mathrm{~km}$

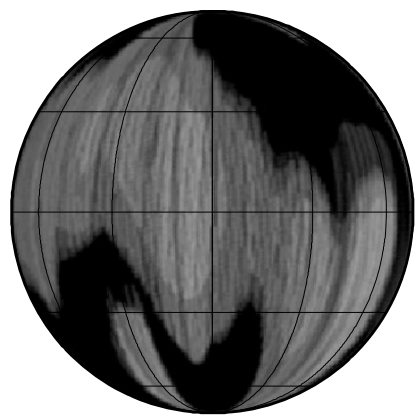

(b) $20-30 \mathrm{~km}$

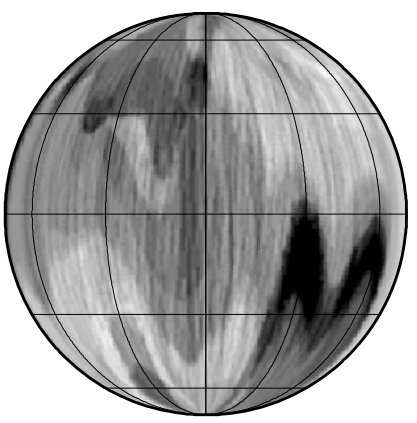

(c) $30-40 \mathrm{~km}$

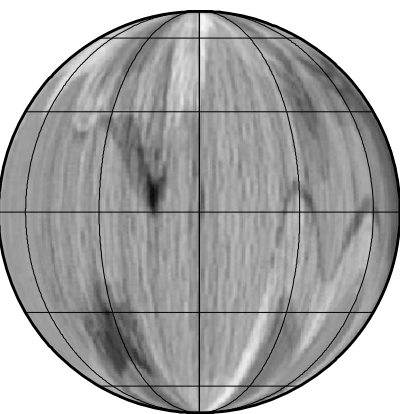

(d) $40-50 \mathrm{~km}$

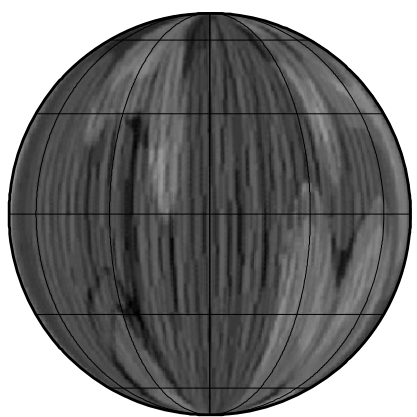

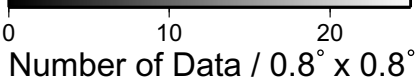

Fig. 3. Density of LOS data for four satellite height ranges.

(a)

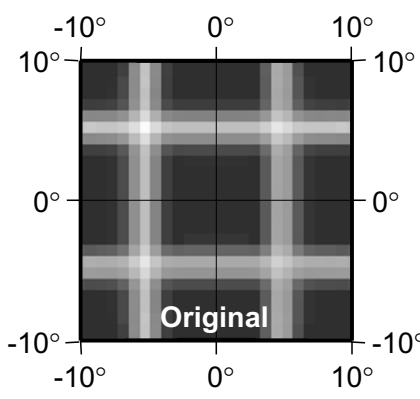

(b)

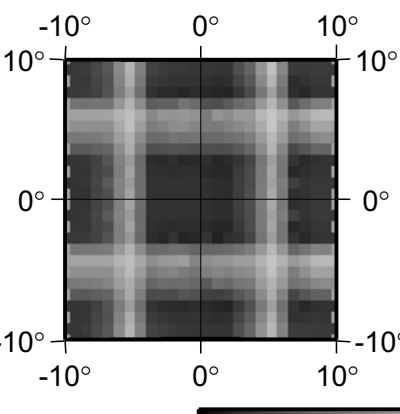

(c)

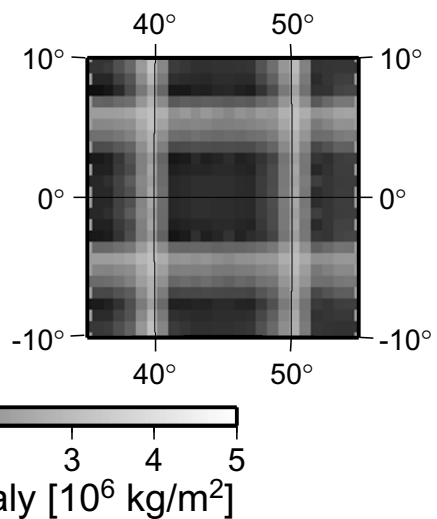

(d)

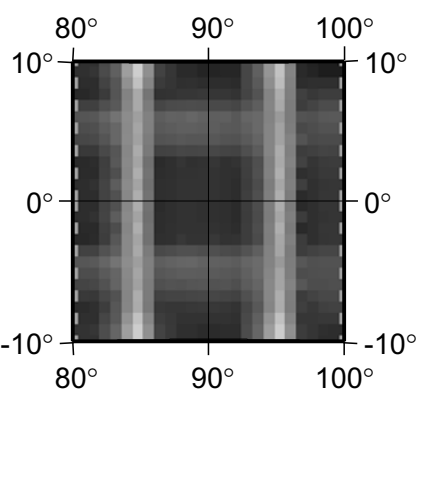

Mass Anomaly $\left[10^{6} \mathrm{~kg} / \mathrm{m}^{2}\right]$

Fig. 4. Results of the inversion test. The original model (a), recovered surface mass anomaly for large blocks $\left(20^{\circ} \times 20^{\circ}\right)$ assumed to be located at different places on the Moon, i.e., $\left(0^{\circ} \mathrm{N}, 0^{\circ} \mathrm{E}\right)(\mathrm{b}),\left(0^{\circ} \mathrm{N}, 45^{\circ} \mathrm{E}\right)(\mathrm{c})$ and $\left(0^{\circ} \mathrm{N}, 90^{\circ} \mathrm{E}\right)(\mathrm{d})$. Spurious results are seen near the large block rim (b)-(d). Also east-west structures are poorly recovered when the satellite is near the lunar near- and farside boundary (d).

The number of data in an inversion run depends on the data density. Typically, we estimate several hundreds of parameters (number of small blocks in a large block) using several tens of thousands of LOS data (number of data points above a large block). Density of LOS data (number of data within small blocks) versus satellite height are shown in Fig. 3. It is found that LOS data obtained at relatively low altitudes $(10-30 \mathrm{~km})$ are not evenly distributed. The accuracy of the estimated mass distribution depends highly on such satellite heights since short-wavelength gravity fields decay rapidly with altitudes.

\subsection{Examination of the Method}

Before applying the method to real LOS data, its performance was tested using synthesized data sets. We consider a $30^{\circ} \times 30^{\circ}$ block (about $900 \mathrm{~km} \times 900 \mathrm{~km}$ ), whose center coincides with the center of a large block. We assume rectangular mountain ranges (about $90 \mathrm{~km}$ wide, $900 \mathrm{~km}$ long and $3 \mathrm{~km}$ high) composed of lunar crustal rocks as dense as $2,900 \mathrm{~kg} \mathrm{~m}^{-3}$, and set two pairs of parallel mountain ranges on the $30^{\circ} \times 30^{\circ}$ block, one pair is striking north-south, and the other pair east-west. The length of mountain ranges was made longer than a large block $\left(20^{\circ} \times 20^{\circ}\right)$ because real topography continues beyond the large block rims. Then we assume real satellite orbits and observation epochs during a certain month out of the LP extended mission, and calculate LOS accelerations coming from the assumed threedimensional topography of the mountain ranges. Such synthesized data sets are prepared at several different longitudes on the lunar equatorial zone, and are used to estimate mass distribution (Fig. 4).

First we notice spurious masses estimated near the large block rims. These are considered to have emerged from topography outside of the large block because we tried to attribute all the gravity signals to the topography within the large block. We also notice that east-west structures are poorly recovered when the satellite is near the lunar nearand farside boundary (Fig. 4(d)). This is because any linear structures parallel with the line-of-sight do not produce acceleration in that direction, i.e. the resolution of gravity field depends on the direction of the structure and the lineof-sight.

Considering the first point, we took out only the central part as large as $10^{\circ} \times 10^{\circ}$ of a large block in order to avoid spurious recovery at the rim, and concatenated them to draw the global map. The second problem is intrinsic to the Earthbased Doppler measurement and will be overcome in fu- 
(a)

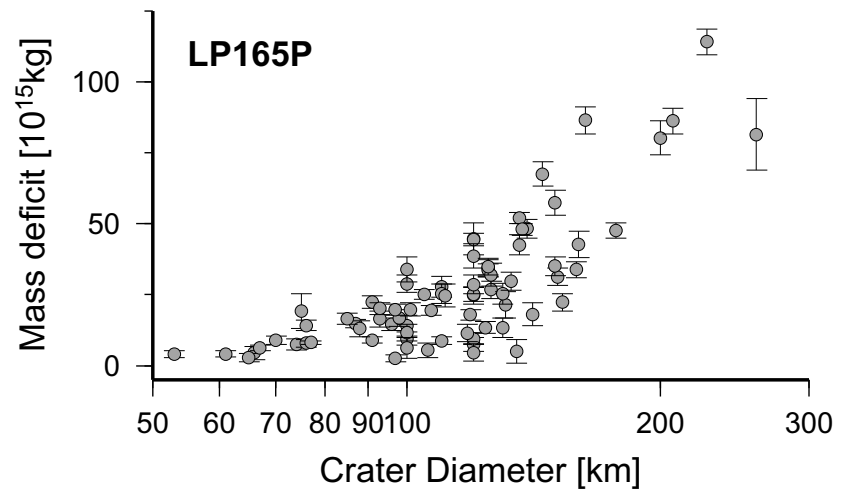

(b)

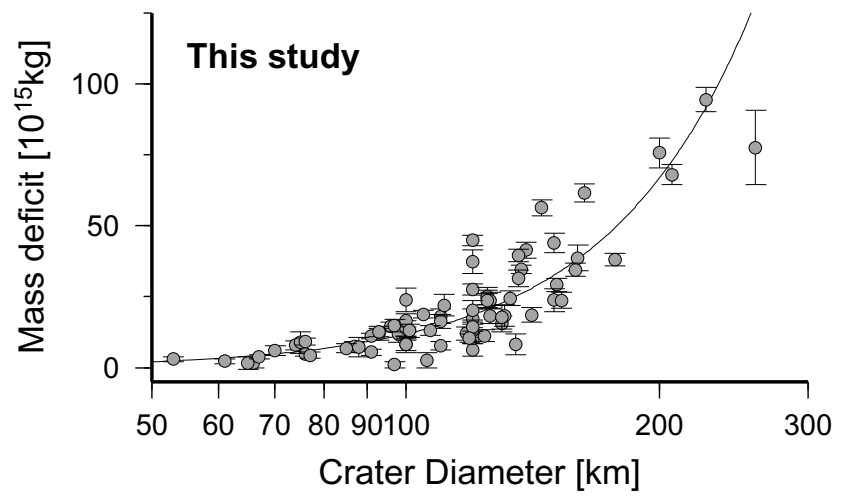

Fig. 5. Mass deficits associated with medium-sized craters from LP165P (a) and LP LOS (this study) (b). The curve denotes the best-fit model (see Table 1).

Table 1. Diameter-mass-deficit relationship for impact craters.

\begin{tabular}{ccccccc}
\hline Method & Planetary Body & Diameter, km & N & Coefficient (a) & Exponent (b) & Source \\
\hline Topography & Moon & $19-150$ & 21 & $6.90 \times 10^{11}$ & 2.31 & Croft (1978) \\
Gravity anomaly & Moon & $50-300$ & 92 & $1.18 \times 10^{11}$ & 2.50 & This study \\
Gravity anomaly & Earth & $<30$ & 4 & N/A & 2.5 & Yokoyama (1974) \\
\hline
\end{tabular}

ture by (1) incorporating very-long-baseline-interferometry (VLBI) tracking data which have sensitivity to the acceleration perpendicular to the LOS direction, and by (2) using Doppler measurements via a relay satellite. Japanese lunar mission SELENE (SELenological and ENgineering Explorer) will realize the VLBI observation of lunar satellites in 2005 (Heki et al., 1999).

\section{Obtained Gravity Anomaly}

Next we processed the real LP LOS to estimate lunar surface mass distribution. In order to compare our results with past studies, we convert the estimated surface mass distribution into gravity anomalies at the lunar surface. Surface density, a surface mass per unit area, $\sigma(\theta, \phi) \mathrm{kg} \mathrm{m}^{-2}$ can be converted into gravity anomaly at the surface $\Delta g \mathrm{~m} \mathrm{~s}^{-2}$ by multiplying $2 \pi G$ (Garland, 1965),

$$
\Delta g(\theta, \phi)=2 \pi G \sigma(\theta, \phi),
$$

where $\theta$ and $\phi$ are the latitude and the longitude, respectively. The obtained gravity anomaly corresponds to free-air gravity anomaly (Fig. 1(b)). Root-mean-squares of the post-fit residuals of the LOS acceleration are around $10 \mathrm{mgal}$ over the entire nearside.

Although total number of estimated parameter is larger than the conventional approach using spherical harmonics, the computation time is relatively short. This is because only small number of parameter (not more than 625) is estimated in one inversion run (i.e., masses on remote points have little inter-parameter correlation, and so stepwise estimation is possible). On the other hand, for the case of LP165P, about 30,000 parameters must be estimated in one inversion run if all the Stokes' coefficients were estimated simultaneously.

Another merit of our method is that we do not need regularization for the inversion. Since direct tracking data of the satellite on the lunar farside cannot be obtained from the Earth, past studies have been relying on numerical techniques to stabilize the solution. For such regularization, the Kaula's rule of thumb has been widely used. In the analysis of the Earth's gravitational potential, Kaula (1963) found that the degree variance of harmonic coefficients is proportional to inverse square of the degree $l$, i.e., $\sim 10^{-5} / l^{2}$. To apply this rule for the Moon, Kaula (1963) used scaling law based on the assumption that the Moon has similar strength to the Earth, and obtained factor of $35.7 \times 10^{-5}$. It is not clear if such a rule is correct and it is controversial how large the factor should be. In fact, Lemoine et al. (1997) used $15 \times 10^{-5}$ for this factor while Konopliv et al. (2001) used $36 \times 10^{-5}$. Our method does not need such constraints to stabilize the solution.

Absolute values of the differences between LP165P (Konopliv et al., 2001) and our gravity anomaly map at the centers of the small blocks are shown in Fig. 1(c). There short wavelength differences are seen all over the nearside. It is also noticed that east-west linear features in LP165P are less conspicuous in our model. They are considered to be short-wavelength noises in the LP165P model. Large differences at the polar regions are thought to be due to the interference between direct signals and those reflected by the lunar surface as suggested by Konopliv et al. (2001). They removed data with observational errors exceeding $\sim 2-$ $3 \mathrm{~mm} \mathrm{~s}^{-1}$ in deriving the LP165P. We did not perform such data screening, and they might have affected our gravity recovery in these regions.

Resolution of the map depends on the size of small blocks. We selected $0.8^{\circ} \times 0.8^{\circ}$, which is comparable to the average height of the satellite during the extended mission. This resolution is equivalent to that of a conventional model using spherical harmonics complete to 225th degree and order. 


\section{Mass Deficits of Medium-Sized Craters}

To exploit high resolution of the gravity anomaly map obtained here, we discuss gravity signatures of craters with diameters $50-300 \mathrm{~km}$. Mass deficit of a crater is obtained as follows, (1) approximate the crater rim with a circle, (2) background surface density is defined as the average of values at points along the circle, (3) surface density $\sigma(\theta, \phi)$ relative to the background are integrated over the interior of the circle, i.e.,

$$
m_{d}=-\int_{S}\left\{\sigma(\theta, \phi)-\sigma_{0}\right\} d s,
$$

where $m_{d}$ is the mass deficit in $\mathrm{kg}, S$ is the area within the circle, and $\sigma_{0}$ is the background surface density. Errors $\delta m_{d}$ shown in Fig. 5 are inferred from the errors of $\sigma_{0}$. Large uncertainties for relatively large craters reflect difficulty in defining $\sigma_{0}$ for such craters (i.e., non uniform density values along the rim). Figure 5 shows the mass deficits of 92 such craters on the lunar nearside as a function of diameter. To compare with the previous work, the mass deficits calculated from LP165P are also shown in Fig. 5(a). Owing to the small spurious gravity features, mass deficits from this work show smaller scatter than those from LP165P (Fig. 5(b)).

Many kinds of quantities $y$ related to lunar impact craters are known to follow,

$$
y=a D^{b},
$$

where $D$ is the crater diameter in $\mathrm{km}$, and $a$ and $b$ are constants (De Hon and Waskom, 1976). For the mass deficits calculated from our gravity anomaly (surface density), we obtained $a=1.18 \times 10^{11} \mathrm{~kg} \mathrm{~km}^{-2.5}$ and $b=2.50$. The model curve is plotted in Fig. 5(b).

For the Earth, using gravity anomaly data, Yokoyama (1974) calculated mass deficits of impact craters with diameter $<30 \mathrm{~km}$, and found they are proportional to 2.5 power of the diameter. Croft (1978) examined lunar crater interior volume (volume deficit) for 21 complex craters with diameter 19-150 km from Lunar Topographic Orthophoto (LTO) maps derived from the Apollo metric photography. The Complex craters are characterized by terraced rims, an uplifted central peak, and flat floor. Croft (1978) obtained $a=0.238$ and $b=2.31$ for complex craters. Assuming crustal density $\rho_{c}$ of $2,900 \mathrm{~kg} \mathrm{~m}^{-3}, a$ for the mass deficits of these craters becomes $6.90 \times 10^{11}$. The relationships between crater diameter and mass deficit of the Earth and the Moon are summarized in Table 1, and our result agrees fairly well with past studies.
High resolution grid topography data from Clementine mission are also available at the PDS web site. Using such data, terrain correction can be performed for the raw LOS data. By conducting the same inversion for the corrected data, we could obtain high resolution terrain-corrected anomaly, i.e., gravity anomaly similar to the Bouguer anomaly which reflects subsurface structures such as the Moho topography. In a future study, this will enable us to discuss compensation of impact craters and elastic thickness of the lunar lithosphere.

Acknowledgments. We thank Yutaka Abe (Univ. Tokyo) for critical review of the original version of the manuscript. This study was carried out at the Mizusawa Astrogeodynamics Observatory, National Astronomical Observatory, Japan.

\section{References}

Bills, B. G. and A. J. Ferrari, A Harmonic analysis of lunar gravity, J. Geophys. Res., 85, 1013-1025, 1980.

Binder, A. B., Lunar prospector: Overview, Science, 281, 1475-1476, 1998.

Croft, S. K., Lunar crater volumes: Interpretation by models of impact cratering and upper crustal structure, Proc. Lunar Planet. Sci. Conf. 9th, 3711-3733, 1978

De Hon, R. A. and J. D. Waskom, Geologic structure of the eastern mare basins, Proc. Lunar. Sci. Conf., 7th, 2769-2746, 1976.

Garland, G. D., The Earth's Shape and Gravity, Pergamon Press Ltd., Headington Hill Hall, Oxford, UK, 1965.

Heki, K., K. Matsumoto, and R. Floberghagen, Three-dimensional tracking of a lunar satellite with differential very-long-baseline-interferometry, Adv. Space Res., 23, 1821-1824, 1999.

Kaula, W. M., The investigation of the gravitational fields of the moon and planets with artificial satellites, Advan. Space Sci. Technol., 5, 210-230, 1963.

Konopliv, A. S., A. B. Binder, L. L. Hood, A. B. Kucinskas, W. L. Sjogren, and J. G. Williams, Improved gravity field of the moon from Lunar Prospector, Science, 281, 476-480, 1998.

Konopliv, A. S., S. W. Asmar, E. Carranza, W. L. Sjogren, and D. N. Yuan, Recent gravity models as a result of the Lunar Prospector mission, Icarus, 150, 1-18, 2001.

Lemoine, F. G. R., D. E. Smith, M. T. Zuber, G. A. Neumann, and D. D. Rowlands, A 70th degree lunar gravity model (GLGM-2) from Clementine and other tracking data, J. Geophys. Res., 102, 16339-16359, 1997.

North, G., Observing the Moon, Cambridge University Press, The Pitt Building, Trumpington Street, Cambridge, UK, 2000.

Simpson, R. A., Software Interface Specification for the Line of Sight Acceleration Profile Data Record (LPSAPDR), 1999.

Williams, J. G., X. X. Newhall, and J. O. Dickey, Lunar moments, tides, orientation, and coordinate frames, Planet. Space Sci., 44, 1077-1080, 1996.

Yokoyama, I., Insekikou ni okeru jyuuryoku sokutei (Gravity measurement at impact craters), in Sokuchigaku no Gaikan (Overview of the Geodesy), edited by The Geodetic Society of Japan, 158 pp., Tokyo office of the Geodetic Society of Japan, 1-3-4 Koishikawa, Bunkyo-ku, Tokyo 112 0002, Japan, 1974

T. Sugano (e-mail: sugano@miz.nao.ac.jp) and K. Heki 